\title{
Assessment of the Latent Adverse Events of Antipsychotic Treatment Using a Subjective Questionnaire in Japanese Patients with Schizophrenia
}

\author{
Masakazu Hatano ${ }^{1,2,3}$, Hiroyuki Kamei ${ }^{3}$, Azusa Kato ${ }^{2}$, Ippei Takeuchi ${ }^{3,4}$, Manako Hanya ${ }^{3}$, Junji Uno ${ }^{4}$, \\ Shigeki Yamada ${ }^{2}$, Kiyoshi Fujita ${ }^{4}$, Nakao Iwata ${ }^{1}$ \\ Departments of ${ }^{1}$ Psychiatry, ${ }^{2}$ Clinical Pharmacy, Fujita Health University School of Medicine, Nagoya, ${ }^{3}$ Office of Clinical Pharmacy Practice and \\ Health Care Management, Faculty of Pharmacy, Meijo University, Nagoya, ${ }^{4}$ Department of Psychiatry, Okehazama Hospital, Toyoake, Japan
}

\begin{abstract}
Objective: The adverse effects of antipsychotic agents can have a marked influence on medication adherence. In this study, we investigated the adverse events of antipsychotics that are less likely to be reported by patients and the reasons why such symptoms remain latent.

Methods: Data were collected by interviewing patients using a subjective questionnaire, and the associations between unreported symptoms and background factors were investigated.

Results: A total of 306 patients with schizophrenia or schizoaffective disorder were examined. Their major symptoms were daytime sleepiness (50.0\%), weight gain (42.2\%), and sexual dysfunction (38.9\%). Sexual dysfunction was nominal significantly more common among the patients that had been treated with antipsychotic agent polypharmacy (odds ratio [OR], 2.14; 95\% confidence interval [Cl], 1.07 to 4.30), and was nominal significantly more common among outpatients (OR, 1.78; 95\% $\mathrm{Cl}, 1.02$ to 3.13). Only approximately $30 \%$ of the patients had reported their symptoms to their physicians.

Conclusion: Patients receiving antipsychotic treatment tolerate some symptoms and do not feel able to report them to their physicians. The most common reason for this is an insufficient patient-physician relationship. Sexual dysfunction is especially hard to identify because it is a delicate problem, and our findings demonstrate that subjective questionnaires are helpful for detecting such symptoms.
\end{abstract}

KEY WORDS: Antipsychotic agents; Adverse drug events; Schizophrenia; Surveys and questionnaires.

\section{INTRODUCTION}

Antipsychotic agents are the most commonly used treatments for schizophrenia. However, more than twothirds of patients discontinue antipsychotic treatment within 18 months. ${ }^{1)}$ Although medication non-adherence can be caused by various factors in patients with schizophrenia, not all of these factors are patient-related problems such as a negative attitude toward medication. Medication-related or environmental problems, including a high antipsychotic dose, severe side effects, and poor therapeutic synergy, can also have a negative influence on medication adherence. ${ }^{2)}$

Extrapyramidal symptoms (EPS) are traditionally con-

Received: June 23, 2016/ Revised: July 26, 2016

Accepted: September 10, 2016

Address for correspondence: Masakazu Hatano, MS Department of Psychiatry, Fujita Health University School of

Medicine, 1-98 Dengakugakubo, Kutsukake, Toyoake, Aichi

470-1192, Japan

Tel: +81-562932157, Fax: +81-562934537

E-mail: hatanomasakazu@yahoo.co.jp sidered to be some of the most severe side effects of antipsychotic treatment. Second generation antipsychotic agents (SGA) are more frequently prescribed than first generation antipsychotic agents (FGA) in the clinical setting because of the reduced risk of EPS. ${ }^{3-5)}$

However, SGA can also induce other types of side effects, e.g., they can cause metabolic syndrome ${ }^{6}$; therefore, physicians need to greater attention to side effect management during the use of such drugs. In addition, physicians are likely to overlook side effects that are not externally visible, such as sexual dysfunction, and patients with such symptoms tend to hesitate to talk about them. Eventually, these symptoms can have a harmful effect on adherence.

Polypharmacy is also associated with non-adherence. In addition to patients simply being unwilling to take a lot of medication, they can also experience side effects because of increased dosages and interactions between antipsychotic agents. ${ }^{7)}$

Although EPS are known to be caused by dopaminergic

(a) This is an Open-Access article distributed under the terms of the Creative Commons Attribution Non-Commercial License (http://creativecommons.org/licenses/by-nc/4.0) which permits unrestricted non-commercial use, distribution, and reproduction in any medium, provided the original work is properly cited. 
blockade $^{8)}$ and the risk of such symptoms increases as the chlorpromazine equivalent value rises, ${ }^{9,10)}$ some other symptoms are caused by different mechanisms ${ }^{7)}$ In addition, antipsychotic drugs are often combined with benzodiazepine or antiparkinson agents. ${ }^{11)}$ Accordingly, the assessment of side effects during antipsychotic treatment has become increasingly complex. In this study, we revealed latent adverse events in patients receiving antipsychotic treatment and investigated their associations with various background factors.

\section{METHODS}

\section{Study Design}

We carried out a subjective survey of schizophrenia patients. The questionnaire used consisted of six questions about adverse events. The first question was whether the patient was suffering from any adverse events at the time. The last of the questions examined the patients' responses to the adverse events such as whether they were tolerating a particular or whether it made them have changed their medicine. Multiple answers were allowed regarding the reasons why the patients tolerated particular symptoms. The questionnaires were conducted by pharmacists to avoid problems about patients being hesitant to talk to their physicians and inaccurate answer by cognitive dysfunction.

In addition, we investigated the associations between the patients' symptoms and various background factors including the dose and number of antipsychotic agents, the main antipsychotic agent, whether the patient was being treated with monotherapy or polypharmacy, whether the patient was receiving combined treatment involving benzodiazepine or antiparkinson agents, and whether the patient was being treated as an inpatient or outpatient. This analysis performed about symptoms were detected in $>30 \%$ of patients. We converted the doses of each antipsychotic agent into chlorpromazine equivalents and classified them into two categories ( $\leq$ or $>600 \mathrm{mg}$ per day). The main antipsychotic agent was defined as the agent with the highest chlorpromazine equivalent value, and comparisons were performed between FGA and SGA. Polypharmacy was defined the prescription of two or more antipsychotic agents. The study was conducted at Fujita Health University Hospital, a general hospital, and six mental hospitals. The study protocol was approved by the ethics committee of Fujita Health University, Japan. The study protocol was adequately explained to all patients, and all of the subjects gave their consent in writing.

\section{Participants}

To be eligible, the patients had to be in a stable condition and to have the ability to answer questions. The patients had all been diagnosed with schizophrenia or schizoaffective disorder according to the definitions outlined in the Diagnostic and Statistical Manual of Mental Disorders, 4th edition, text revision (DSM-IV-TR), and had taken at least one antipsychotic agent.

\section{Statistical Analysis}

The associations between adverse events and each background factor were analyzed using a logistic regression model including the following covariates: age, sex, the dose and number of antipsychotic agents, the main antipsychotic agent, combination therapy involving benzodiazepine or antiparkinson agents, and whether the patient was an inpatient or outpatient. The effect of the type of the main antipsychotic agent; i.e., whether it was an FGA or SGA, was also assessed. The analyzed SGA included risperidone, olanzapine, and aripiprazole, which the top three most commonly prescribed antipsychotic agents. $p$ values of $<0.05$ before Bonferroni correction were considered as nominally significant. After applying a Bonferroni correction for multiple testing, which resulted in a $p$ values of $<0.0083(0.05 / 6)$. Statistical analyses were performed with EZR (Saitama Medical Centre, Jichi Medical University; http://www.jichi.ac.jp/saitama-sct/SaitamaHP.files/ statmedEN.html), which is a

Table 1. Patient characteristics

\begin{tabular}{lc}
\hline \multicolumn{1}{c}{ Characteristic } & Data \\
\hline Age (yr) & $47.9 \pm 14.2$ \\
Male sex (\%) & 52.3 \\
Main antipsychotic agent (\%) & \\
Risperidone & 47.1 \\
Olanzapine & 18.6 \\
Aripiprazole & 11.8 \\
Quetiapine & 7.2 \\
Perospirone & 4.6 \\
Blonanserin & 2.0 \\
First generation antipsychotics & 8.8 \\
Chlorpromazine equivalents (mg) & $548.3 \pm 427.6$ \\
Diazepam equivalents (mg) & $2.0 \pm 2.8$ \\
Biperiden equivalents (mg) & $0.5 \pm 1.0$ \\
Polypharmacy (\%) & 34.3 \\
Combined use of other agents (\%) & \\
Benzodiazepine & 61.1 \\
Antiparkinson agents & 31.0 \\
Therapeutic system (\%) & \\
Outpatients & 55.2 \\
Inpatients & 44.8 \\
\hline
\end{tabular}


graphical user interface for $\mathrm{R}$ (version 2.13.2; The $\mathrm{R}$ Foundation for Statistical Computing, Vienna, Austria). More precisely, it is a modified version of $\mathrm{R}$ commander (version 1.8-4) designed to add statistical functions that are frequently used in biostatistics. ${ }^{12)}$

\section{RESULTS}

\section{Patient Characteristics}

Between December 2009 and July 2010, a total of 306 patients participated (males, 160; females, 146; age [mean \pm standard deviation], $47.9 \pm 14.2$ years) in this study. The patients' characteristics are summarized in Table 1. Risperidone was the main antipsychotic agent in almost half of cases $(n=144)$. The mean chlorpromazine equivalents dose was $548.3 \pm 427.6 \mathrm{mg}$ per day, and the prevalence of monotherapy was $65.7 \%(n=201)$. The frequencies of combined treatment involving benzodiazepine or antiparkinson agents were $61.1 \%$ and $31.0 \%$, respectively.

\section{Questionnaire}

All patients had at least one symptom, and the mean number of symptoms was 3.5. The results of the questionnaire about these five symptoms are shown in Table 2. The most common symptom was daytime sleepiness $(\mathrm{n}=153,50.0 \%)$. Weight gain, sexual dysfunction, akathisia, and dry mouth were detected in $>30 \%$ of patients. Regarding the proportions of patients that considered these symptoms to be side effects of their drug treatment, dry mouth $(\mathrm{n}=53,53.0 \%)$ was most commonly considered to be a side effect of treatment, and almost all symptoms were considered to be side effects by $<50 \%$ of the patients. Only a few patients had changed their medication due to adverse effects $(n=45,4.1 \%)$. As for the proportions of patients that tolerated the five symptoms, symptom tolerance was seen in $>30 \%$ of patients that exhibited weight gain or sexual dysfunction. Although daytime sleepiness was the most common complaint, the frequency of patients who tolerated this symptom was lower $(n=21,13.7 \%)$ than the values for the other symptoms. Approximately $30 \%$ of the patients had reported their symptoms to their physicians. The most common reason for tolerating the five symptoms was "the physician did not listen to my complaints" ( $\mathrm{n}=137,51.3 \%)$, and this response was seen at a particularly high rate for sexual dysfunction ( $\mathrm{n}=22,61.1 \%)$. The second most common reason was "I recognized the necessity of controlling the symptom" ( $\mathrm{n}=123,46.1 \%)$.

\section{Associations between Adverse Events and Background Factors}

Table 3 shows the associations between adverse events and various background factors. There was no significant relationship between the frequency of each adverse event and the antipsychotic dose. Regarding the main antipsychotic agent, the frequencies of the adverse events did not differ significantly between FGA and SGA. On the other hand, polypharmacy was associated with a nominally significant higher risk of sexual dysfunction (odds ratio [OR], 2.14; 95\% confidence interval [CI], 1.07 to $4.30 ; p<0.05)$. Combination therapy involving benzodiazepine was associated with a significantly higher risk of akathisia (OR, 2.36; 95\% CI, 1.29 to $4.32 ; p<0.0083$ ), and nominally significant higher risk of the total number of checkups (beta, $0.65 ; 95 \% \mathrm{CI}, 0.11$ to $1.20 ; p<0.05$ ).

Table 2. Results of questionnaire about the top five symptoms

\begin{tabular}{|c|c|c|c|c|c|c|}
\hline \multirow[b]{2}{*}{ Items } & \multicolumn{6}{|c|}{ Yes (\%) } \\
\hline & $\begin{array}{l}\text { Daytime } \\
\text { sleepiness }\end{array}$ & $\begin{array}{l}\text { Weight } \\
\text { gain }\end{array}$ & $\begin{array}{c}\text { Sexual } \\
\text { dysfunction }\end{array}$ & Akathisia & $\begin{array}{c}\text { Dry } \\
\text { mouth }\end{array}$ & Total \\
\hline 1. I have this symptom. & $153 / 306(50.0)$ & $129 / 306(42.2)$ & $119 / 306(38.9)$ & $104 / 306(34.0)$ & $100 / 306(32.7)$ & $1,092 / 306(357)$ \\
\hline 2. I consider this symptom to be a side effect. & $69 / 153(45.1)$ & $35 / 129(27.1)$ & $46 / 119(38.7)$ & $25 / 104(24.0)$ & $53 / 100(53.0)$ & $396 / 1,092(36.3)$ \\
\hline 3. I have changed my medication because of this symptom. & $8 / 153(5.2)$ & $6 / 129(4.7)$ & $3 / 119(2.5)$ & $7 / 104(6.7)$ & $1 / 100(1.0)$ & $45 / 1,092(4.1)$ \\
\hline 4. I have tolerated this symptom. & $21 / 153(13.7)$ & $41 / 129(31.8)$ & $36 / 119(30.3)$ & $25 / 104(24.0)$ & $27 / 100(27.0)$ & $267 / 1,092(24.5)$ \\
\hline $\begin{array}{l}\text { 5. I have reported this symptom to my physician. } \\
\text { 6. Reasons for symptom tolerance. (multiple answers allowed) }\end{array}$ & $38 / 153(24.8)$ & $45 / 129(34.9)$ & $28 / 119(23.5)$ & $38 / 104(36.5)$ & $17 / 100(17.0)$ & $323 / 1,092(29.6)$ \\
\hline 6-1. It is difficult to talk to the physician. & $5 / 21(23.8)$ & $12 / 41(29.3)$ & $21 / 36(58.3)$ & $5 / 25(20.0)$ & $1 / 27(3.7)$ & $80 / 267(30.0)$ \\
\hline 6-2. The physician did not listen to my complaints & $10 / 21(47.6)$ & $20 / 41(48.8)$ & $22 / 36(61.1)$ & $8 / 25(32.0)$ & $13 / 27(48.1)$ & $137 / 267(51.3)$ \\
\hline 6-3. I have no time to talk about this symptom. & $2 / 21(9.5)$ & $3 / 41(7.3)$ & $3 / 36(8.3)$ & $5 / 25(20.0)$ & $3 / 27(11.1)$ & $29 / 267(10.9)$ \\
\hline 6-4. I feel guilty for the physician. & $3 / 21(14.3)$ & $7 / 41(17.1)$ & $7 / 36(19.4)$ & $2 / 25(8.0)$ & $1 / 27(3.7)$ & $43 / 267(16.1)$ \\
\hline 6-5. I am scared to report this symptom. & $1 / 21(4.8)$ & $4 / 41(9.8)$ & $2 / 36(5.6)$ & $3 / 25(12.0)$ & $1 / 27(3.7)$ & $26 / 267(9.7)$ \\
\hline 6-6. I am pleased with my current medication. & $6 / 21(28.6)$ & $16 / 41(39.0)$ & $8 / 36(22.2)$ & $9 / 25(36.0)$ & $6 / 27(22.2)$ & $79 / 267(29.6)$ \\
\hline 6-7. I consider it necessary to control my symptoms. & $11 / 21(52.4)$ & $10 / 41(24.4)$ & $21 / 36(58.3)$ & $13 / 25(52.0)$ & $9 / 27(33.3)$ & $123 / 267(46.1)$ \\
\hline 6-8. I feel changing my medicine will make no difference. & $5 / 21(23.8)$ & $10 / 41(24.4)$ & $13 / 36(36.1)$ & $4 / 25(16.0)$ & $8 / 27(29.6)$ & $81 / 267(30.3)$ \\
\hline 6-9. My medicine will be increased if I report this symptom. & $2 / 21(9.5)$ & $4 / 41(9.8)$ & $6 / 36(16.7)$ & $2 / 25(8.0)$ & $2 / 27(7.4)$ & $39 / 267(14.6)$ \\
\hline
\end{tabular}


Table 3. Associations between adverse events and patient background factors

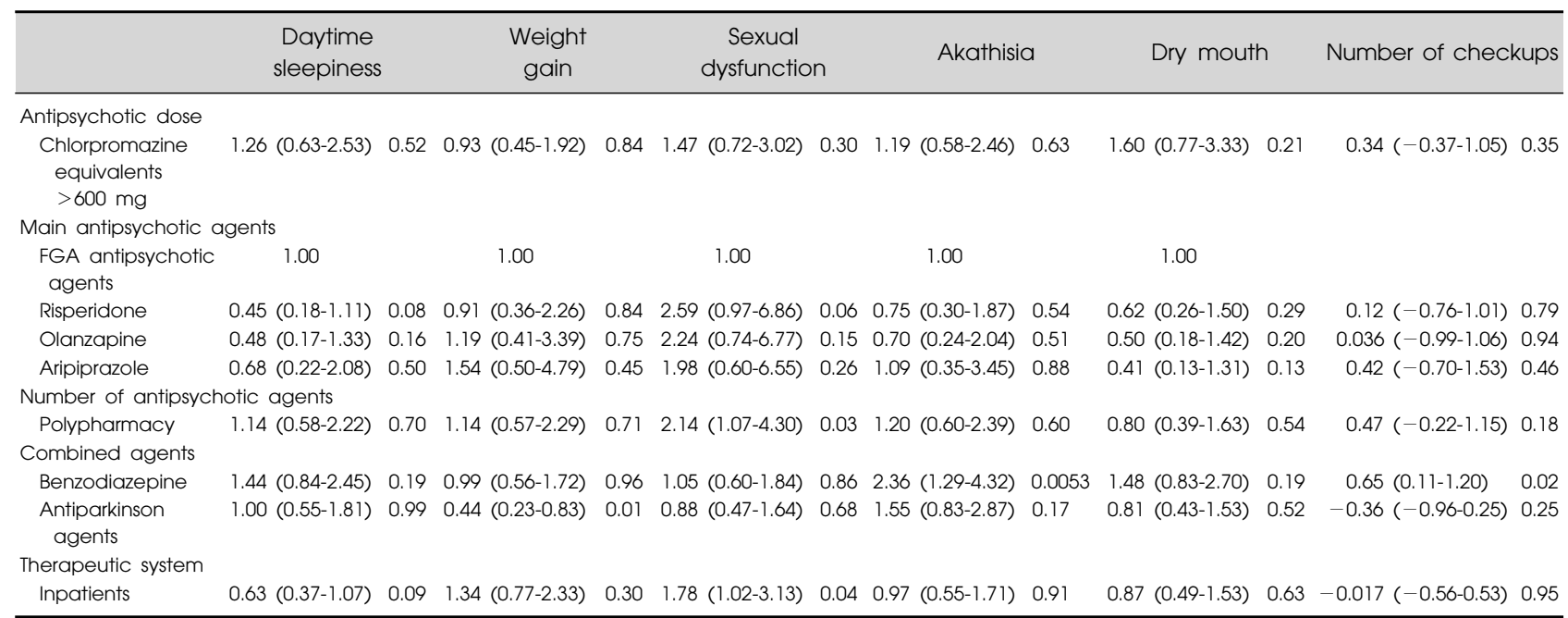

Values are presented as odds ratio ( $95 \%$ confidence interval) and $p$ value.

Odds ratios are adjusted for age, sex, the dose and number of antipsychotic agents, the type of the main antipsychotic agent, the use of combined treatment involving benzodiazepine or antiparkinson agents, and the therapeutic system.

FGA, first generation antipsychotic agent.

Combination therapy involving antiparkinson agents was associated with a nominally significant lower risk of weight gain (OR, $0.44 ; 95 \% \mathrm{CI}, 0.23$ to $0.83 ; p<0.05$ ). Finally, outpatients were found to be at a nominally significant higher risk of sexual dysfunction than inpatients (OR, 1.78; 95\% CI, 1.02 to 3.13; $p<0.05$ ). However, the differences were not significant after the Bonferroni correction was applied other than the akathisia with benzodiazepine.

\section{DISCUSSION}

In the present study, we carried out a subjective survey to reveal adverse events of antipsychotic treatment that are often not reported to physicians. The patients most commonly complained of the following symptoms (in order, high to low frequency): daytime sleepiness, weight gain, sexual dysfunction, akathisia, and dry mouth. On the other hand, except for akathisia, EPS, such as tremors and sialorrhea, exhibited low frequencies. These results correspond with those of a previous questionnaire-based study. ${ }^{13)}$ One possible reason for this finding is that physicians tend to prescribe antiparkinson agents and decrease the doses of antipsychotic agents as soon as EPS develop. All of the patients had at least one symptom; thus, our results suggest that the patients might have been suffering from adverse events that their physicians were not aware of. Obviously, not all symptoms are side effects of antipsychotic treatment. This problem is the greatest limitation of this study. However, it is evident that many of the patients in this study tolerated certain symptoms and did not feel able to report them to their physicians. In addition, the main reason why the patients tolerated these symptoms was that "the physician did not listen to my complaints". Therefore, our study demonstrated that an insufficient patient-physician relationship causes symptoms to go unreported. This subjective questionnaire has the potential to assist in revealing such adverse events.

The most common symptoms do not always become a major problem for patients. Although daytime sleepiness was the most common symptom complained of by the patients in the current study, the proportion of patients that said they tolerated it tended to be lower than for other symptoms. As for weight gain and sexual dysfunction, over $30 \%$ of patients tolerated these symptoms, and the frequency of symptom tolerance was particularly high for sexual dysfunction. In addition, the proportion of patients who answered "I have reported this symptom to my physician" was lower for sexual dysfunction. The main reason for this was considered to be that patients feel ashamed to report sexual dysfunction to their physician. In addition, gender differences between the patient and physician can cause communication difficulties. Moreover, outpatients were found to be at a nominal significantly higher risk of sexual dysfunction than inpatients. Thus, our results suggest that interventions involving subjective tools are particularly important for assessing outpatients.

The frequency of sexual dysfunction was estimated to range from $30-80 \%$ of patients with schizophrenia in pre- 
vious systematic studies. ${ }^{14)}$ One study reported that patients under 30 years of age exhibit a significantly higher rate of sexual dysfunction. ${ }^{15)}$ Based on these findings, our study detected a relatively low rate of sexual dysfunction. This might have been because we analyzed a larger number of elderly patients. Although no significant difference in the frequency of sexual dysfunction was detected between the patients treated with FGA and SGA in this study, in general the frequency and severity of hyperprolactinemia, which is the main cause of antipsychoticinduced sexual dysfunction, depends on the strength of dopamine D2 receptor blockade. ${ }^{16)}$ In fact, a previous review summarized the relative impact of the sexual dysfunction induced by various antipsychotics as follows: risperidone $>$ FGA (haloperidol) $>$ olanzapine $>$ quetiapine $>$ aripiprazole. ${ }^{17)}$ We consider that our results can be explained by the fact that various mechanisms other than dopamine D2 receptor blockade are associated with antipsychotic-induced sexual dysfunction and the sample size for each antipsychotic agent was too small. A previous study reported that sexual dysfunction is triggered by histaminergic, cholinergic, serotonergic, and adrenergic blockade, ${ }^{18)}$ and basic research has suggested that the plasma concentration of prolactin is not associated with sexual dysfunction. ${ }^{19)}$ Moreover, a recent study of bipolar disorder reported that polypharmacy is a risk factor for sexual dysfunction. ${ }^{20)}$ In our study, sexual dysfunction was found to be nominal significantly related to polypharmacy, but not to the dose of antipsychotic agents. These results suggest that it is difficult to predict sexual dysfunction based on chlorpromazine equivalents.

Our study had several limitations. Not all of the symptoms identified by our subjective questionnaire were side effects of antipsychotic treatment, as mentioned above. For example, akathisia had a potential for not side effect but psychiatric symptom. Thus, although this survey indicated that combined treatment involving benzodiazepines increase risk of akathisia, it is also suspected that benzodiazepines used to control the symptom. Furthermore, our study did not assess the severity of the patients' disease or side effects, the duration of episode, the age of onset, the cognitive function, the employment and marital status, medication adherence, or the duration of antipsychotic treatment. In addition, the significant result was only the akathisia with benzodiazepine. The most conservative for such multiple comparison would apply an adjusted $p$ values of $<0.0083$, since we performed the analyses under top five symptom and number of checkups (resulting six tests per dataset). Therefore, the results ob- tained should be interpreted with caution.

In conclusion, the present study revealed that patients that were being treated with antipsychotic drugs had various latent symptoms. Sexual dysfunction is especially hard to notice because it is a delicate problem, and our findings demonstrate that subjective questionnaires are helpful for detecting such symptoms. We expect that the use of tools like the questionnaire employed in this study by clinicians will help to improve treatments for schizophrenia.

\section{REFERENCES}

1. Lieberman JA, Stroup TS, McEvoy JP, Swartz MS, Rosenheck RA, Perkins DO, et al. Effectiveness of antipsychotic drugs in patients with chronic schizophrenia. $N$ Engl J Med 2005;353:1209-1223.

2. Lacro JP, Dunn LB, Dolder CR, Leckband SG, Jeste DV. Prevalence of and risk factors for medication nonadherence in patients with schizophrenia: a comprehensive review of recent literature. J Clin Psychiatry 2002;63:892-909.

3. Marder SR, Meibach RC. Risperidone in the treatment of schizophrenia. Am J Psychiatry 1994;151:825-835.

4. Fujimaki K, Takahashi T, Morinobu S. Association of typical versus atypical antipsychotics with symptoms and quality of life in schizophrenia. PLoS One 2012;7:e37087.

5. Zhang JP, Gallego JA, Robinson DG, Malhotra AK, Kane JM, Correll CU. Efficacy and safety of individual secondgeneration vs. first-generation antipsychotics in first-episode psychosis: a systematic review and meta-analysis. Int $J$ Neuropsychopharmacol 2013;16:1205-1218.

6. DE Hert M, Correll CU, Bobes J, Cetkovich-Bakmas M, Cohen D, Asai I, et al. Physical illness in patients with severe mental disorders. I. Prevalence, impact of medications and disparities in health care. World Psychiatry 2011;10: 52-77.

7. Gallego JA, Nielsen J, De Hert M, Kane JM, Correll CU. Safety and tolerability of antipsychotic polypharmacy. Expert Opin Drug Saf 2012;11:527-542.

8. Kapur S, Zipursky R, Jones C, Remington G, Houle S. Relationship between dopamine $D(2)$ occupancy, clinical response, and side effects: a double-blind PET study of firstepisode schizophrenia. Am J Psychiatry 2000;157:514-520.

9. Geddes J, Freemantle N, Harrison P, Bebbington P. Atypical antipsychotics in the treatment of schizophrenia: systematic overview and meta-regression analysis. BMJ 2000;321: 1371-1376.

10. Ezewuzie N, Taylor D. Establishing a dose-response relationship for oral risperidone in relapsed schizophrenia. J Psychopharmacol 2006;20:86-90.

11. Shinfuku M, Uchida H, Tsutsumi C, Suzuki T, Watanabe $\mathrm{K}$, Kimura Y, et al. How psychotropic polypharmacy in schizophrenia begins: a longitudinal perspective. Pharmacopsychiatry 2012;45:133-137.

12. Kanda Y. Investigation of the freely available easy-to-use software 'EZR' for medical statistics. Bone Marrow Transplant 2013;48:452-458.

13. Hashimoto Y, Uno J, Miwa T, Kurihara M, Tanifuji H, Tensho M. Effects of antipsychotic polypharmacy on sideeffects and concurrent use of medications in schizophrenic outpatients. Psychiatry Clin Neurosci 2012;66:405-410.

14. Park YW, Kim Y, Lee JH. Antipsychotic-induced sexual dysfunction and its management. World J Mens Health 
2012;30:153-159.

15. Fujii A, Yasui-Furukori N, Sugawara N, Sato Y, Nakagami $\mathrm{T}$, Saito M, et al. Sexual dysfunction in Japanese patients with schizophrenia treated with antipsychotics. Prog Neuropsychopharmacol Biol Psychiatry 2010;34:288-293.

16. Wieck A, Haddad PM. Antipsychotic-induced hyperprolactinaemia in women: pathophysiology, severity and consequences. Selective literature review. $\mathrm{Br} J$ Psychiatry 2003; 182:199-204.

17. Baggaley M. Sexual dysfunction in schizophrenia: focus on recent evidence. Hum Psychopharmacol 2008;23:201-209.

18. Malik P. Sexual dysfunction in schizophrenia. Curr Opin
Psychiatry 2007;20:138-142.

19. Yasui-Furukori N, Fujii A, Sugawara N, Tsuchimine S, Saito M, Hashimoto $\mathrm{K}$, et al. No association between hormonal abnormality and sexual dysfunction in Japanese schizophrenia patients treated with antipsychotics. Hum Psychopharmacol 2012;27:82-89.

20. Brooks JO 3rd, Goldberg JF, Ketter TA, Miklowitz DJ, Calabrese JR, Bowden CL, et al. Safety and tolerability associated with second-generation antipsychotic polytherapy in bipolar disorder: findings from the Systematic Treatment Enhancement Program for Bipolar Disorder. J Clin Psychiatry 2011;72:240-247. 